\title{
A NOTE ON INTEGRATION OF VECTOR VALUED FUNCTIONS
}

\author{
R. B. DARST
}

I. Introduction. The Riesz representation theorem and the Hildebrandt-Fichtenholz-Kantorovitch (H-F-K) theorem establish that the adjoint spaces of certain spaces of continuous real valued functions are isomorphic and isometric to certain spaces of functions of bounded variation or spaces of finitely additive set functions. Moreover, in the setting of the Riesz theorem, there are the following dual characterizations of each of the associated spaces in terms of the other.

(a) If $f$ is a real valued function on the interval $[0,1]$, then $f$ is continuous on $[0,1]$ if, and only if, the Stieltjes integral $\int_{0}^{1} f d g$ exists for each real valued function $g$ of bounded variation on $[0,1]$.

(b) If $g$ is a real valued function on $[0,1]$, then $g$ is of bounded variation on $[0,1]$ if, and only if, the Stieltjes integral $\int_{0}^{1} f d g$ exists for each continuous function $f$ on $[0,1]$.

The H-F-K type analog of (a) has been established by Leader [8], and we shall establish the corresponding analog of (b) in the course of this note. However, the primary purpose of this note is to discuss more general H-F-K type analogs of (a) and (b). For an excellent survey and extensive bibliography of related results, we refer the reader to [7].

II. Notation and terminology. Let $X$ be a set, $S$ be an algebra of subsets of $X$, and each of $B$ and $B_{1}$ be a nontrivial Banach space. We denote by

$\chi(E)$ the characteristic function of the subsets $E$ of $X$,

$C=C(X, S, B)$ the Banach space (sup norm) of $B$-valued functions on $X$ that are continuous with respect to $S$ (cf. $[1 ; 4])$ : if $\epsilon>0$ then there exists a partition $\pi$ of $X$ in $S$ (by a partition, we mean a finite partition and by a partition $\pi$ in $S$, we mean that $\pi \subset S$ ) such that $\sup _{E \in \pi} O(f, E)<\epsilon$, where $O(f, E)=\sup _{x, y \in E}\|f(x)-f(y)\|$,

$H(X, S)$ the Banach space (variation norm) of bounded and finitely additive set functions (real valued) on $S$,

$H\left(B_{2}\right)=H\left(X, S, B_{2}\right)$ the Banach space (cf. [2]) of bounded and finitely additive functions $G$ on $S$ to a Banach space $B_{2}$ :

(1) $G(\theta)=0$,

(2) if $E, F \in S$ and $E \cap F=\theta$, then $G(E \cup F)=G(E)+G(F)$, and

(3) $\|G\|=\sup \left[\sum_{E \in \pi}\|G(E)\| ; \pi\right.$ is a partition of $X$ in $\left.S\right]$,

Received by the editors November 6, 1961. 
$\left(B, B_{1}\right)$ the Banach space (uniform operator norm; cf. [3, p. 475]) of bounded homomorphisms on $B$ to $B_{1}$,

$W=W\left(X, S,\left(B, B_{1}\right)\right)$ the Banach space of weakly bounded and finitely additive set functions $G$ on $S$ to $\left(B, B_{1}\right)$ where the weak norm $\|G\|_{w}$ of $G$ is $\sup \left[\left\|\sum_{i \leqq n} G\left(E_{i}\right)\left(x_{i}\right)\right\| ; \pi=\left[E_{i} ; i \leqq n\right]\right.$ is a partition of $X$ in $S$ and $\left.x_{i} \in B_{u}=[x \in B ;\|x\| \leqq 1]\right]$,

$\int_{X} f d g$ the Stieltjes integral (cf. [6]) of a $B$-valued function $f$ on $X$ with respect to a finitely additive set function $g$ on $S$, and

$\int_{X} d G \cdot f$ the Stieltjes integral (cf. [2]: $\int_{X} d G \cdot f=\lim _{\pi}\left[\sum_{i \leq n} G\left(E_{i}\right)\right.$ . $\left.\left(f\left(x_{i}\right)\right) ; \pi=\left[E_{i} ; i \leqq n\right], x_{i} \in E_{i}\right]$ where the limit is taken in the MooreSmith sense over the directed set of partitions $\pi$ of $X$ in $S$ (cf. [6])) of a $B$-valued function $f$ on $X$ with respect to a $\left(B, B_{1}\right)$-valued finitely additive function $G$ on $S$.

Before proceeding to the text of this note, we wish to establish the following

Lemma. If $G \in W$, then $\|G\|_{w}=\sup \left[\left\|\sum_{i \leqq n} G\left(E_{i}\right)\left(x_{i}\right)\right\| ;\left[E_{i} ; i \leqq n\right]\right.$ is a subset of a partition of $X$ in $S$ and $\left\|x_{i}\right\|=1$ for $\left.i \leqq n\right]$.

Proof. In order to prove this lemma, let us recall the following fact. If each of $u$ and $v$ is an element of a Banach space and $0 \leqq \lambda \leqq 1$, then $\|u+\lambda v\| \leqq \max [\|u\|,\|u+v\|]:\|u+\lambda v\|=\|(1-\lambda) u+\lambda(u+v)\|$ $\leqq(1-\lambda)\|u\|+\lambda\|u+v\|$. Now let $\left[F_{i} ; i \leqq m\right]$ be a partition of $X$ in $S$, $x_{i} \in B_{u}, x_{p} \neq 0$ and $\lambda=\left\|x_{p}\right\|$. Then

$$
\begin{aligned}
& \left\|\sum_{i \leqq m} G\left(F_{i}\right)\left(x_{i}\right)\right\| \\
& =\left\|\sum_{i \neq p} G\left(F_{i}\right)\left(x_{i}\right)+\lambda G\left(F_{p}\right)\left(x_{p} /\left\|x_{p}\right\|\right)\right\| \\
& \leqq \max \left[\left\|\sum_{i \neq p} G\left(F_{i}\right)\left(x_{i}\right)\right\|,\left\|\sum_{i \neq p} G\left(F_{i}\right)\left(x_{i}\right)+G\left(F_{p}\right)\left(x_{p} /\left\|x_{p}\right\|\right)\right\|\right] .
\end{aligned}
$$

III. Representation theorem and remarks. In order to motivate the ensuing discussion, we shall begin this section by stating a fairly general form of the Hildebrandt-Fichtenholz-Kantorovitch theorem.

THEOREM 1. The space of bounded homomorphisms $T$ on $C$ to $B_{1}$ is isomorphic and isometric to $W$; there exists, uniquely, $G \in W$ such that (1) if $f \in C$, then $T(f)=\int_{X} d G \cdot f$ and (2) $\|G\|_{w}=\|T\|$.

If $B_{1}$ is the space of real numbers, then $\left(B, B_{1}\right)=B^{*}$ and, moreover, $W=H\left(B^{*}\right)$; i.e., $W$ and $H\left(B^{*}\right)$ are composed of the same set functions and the norms are identical. (If $B_{1}$ is finite dimensional, the ele- 
ments of $W$ are strongly bounded and $W$ is equivalent to $H\left(\left(B, B_{1}\right)\right)$.) A discussion of the case where $W=H\left(B^{*}\right)$ can be found in [2]. There is an extensive discussion of results of this type in [3].

Turning now to our professed purpose.

LEMMA 1. Let $G$ be a finitely additive set function on $S$ to $\left(B, B_{1}\right)$ that is not weakly bounded and $\gamma>0$. Then there exists a partition $\left[E_{i} ; i \leqq n\right]$ of $X$ in $S$ and a finite subset $\left[y_{i} ; i<n\right]$ of $B_{u}$ such that $\left\|\sum_{i<n} G\left(E_{i}\right)\left(y_{i}\right)\right\|$ $>\gamma$ and the restriction $G / E_{n}$ of $G$ to $E_{n}$ is not weakly bounded.

Proof. There exists a partition $\left[E_{i} ; i \leqq n\right]$ of $X$ in $S$ and a subset $\left[y_{i} ; i \leqq n\right]$ of $B_{u}$ such that

$$
\left\|\sum_{i \leqq n} G\left(E_{i}\right)\left(y_{i}\right)\right\|>\|G(X)\|+2 \gamma
$$

and

$$
G / E_{n}
$$

is not weakly bounded. Hence

$$
\begin{aligned}
\left\|\sum_{i<n} G\left(E_{i}\right)\left(y_{i}\right)\right\| & >\|G(X)\|+2 \gamma-\left\|G\left(E_{n}\right)\left(y_{n}\right)\right\| \\
& \geqq\|G(X)\|+2 \gamma-\left\|G\left(E_{n}\right)\right\| .
\end{aligned}
$$

Let

$$
\Re=\sup \left[\left\|\sum_{i<n} G\left(E_{i}\right)\left(x_{i}\right)\right\| ;\left[x_{i} ; i<n\right] \subset B_{u}\right]
$$

Then

$$
\mathfrak{N} \geqq\left\|\sum_{i<n} G\left(E_{i}\right)\left(y_{i}\right)\right\|>\|G(X)\|+2 \gamma-\left\|G\left(E_{n}\right)\right\| .
$$

Moreover,

$$
\Re \geqq \sup \left[\left\|\sum_{i<n} G\left(E_{i}\right)(x)\right\| ; x \in B_{u}\right]=\left\|G\left(\bigcup_{i<n} E_{i}\right)\right\|
$$

(recall that $\sum_{i<n} G\left(E_{i}\right)=G\left(\bigcup_{i<n} E_{i}\right)$ ) and, hence,

$$
\Re \geqq\left\|G\left(\bigcup_{i<n} E_{i}\right)\right\| \geqq\left\|G\left(E_{n}\right)\right\|-\|G(X)\| .
$$

Finally, adding (4) and (5), we have $\Re>\gamma$. 
THEOREM 2. Let $G$ be a finitely additive function on $S$ to $\left(B, B_{1}\right)$. Then $G$ is weakly bounded if, and only if, the Stieltjes integral $\int_{X} d G \cdot f$ exists for each $f \in C$.

Proof. Suppose $\|G\|_{w}<\infty, f \in C$, and $\epsilon>0$. Let $\pi$ be a partition of $X$ in $S$ such that $\sup _{E \in \pi} 0(f, E)<\epsilon /\|G\|_{w}$. Then any two refinement sums of $\pi$ differ by less than $\epsilon$ and, hence, $\int_{X} d G \cdot f$ exists.

Suppose $\|G\|_{w}=\infty$. Then, by Lemma 1 , there exists a partition $\left[E_{1 i} ; i \leqq n_{1}\right]$ of $X$ in $S$ and a subset $\left[x_{1 i} ; i<n_{1}\right]$ of $B_{u}$ such that $\left\|\sum_{j<n_{1}} G\left(E_{1 j}\right)\left(x_{1 j}\right)\right\|>1$ and $G / E_{1_{1} n_{1}}$ is not weakly bounded. Applying Lemma 1 to $G / E_{1 n_{1}}$, there exists a partition $\left[E_{2 i} ; i \leqq n_{2}\right]$ of $E_{1 n_{1}}$ and a subset $\left[x_{2 i} ; i<n_{2}\right]$ of $B_{u}$ such that $\left\|\sum_{j<n_{2}} G\left(E_{2 j}\right)\left(x_{2 j}\right)\right\|>2^{2}$ and $G / E_{2 n_{2}}$ is not weakly bounded. Hence, by induction, there exists a sequence $\left\{\left[E_{i j} ; j<n_{i}\right]\right\}$ of finite subsets of $S$ and a sequence $\left\{\left[x_{i j} ; j<n_{i}\right]\right\}$ of subsets of $B_{u}$ such that (1) $\left(\bigcup_{j<n_{i}} E_{i j}\right) \cap\left(\bigcup_{j<n_{k}} E_{k j}\right)=\varnothing$ if $i \neq k$ and (2) $\left\|\sum_{j<n_{i}} G\left(E_{i j}\right)\left(x_{i j}\right)\right\|>i^{2}$. Let $f=\sum_{i \geqq 1} i^{-1}\left\{\sum_{j<n_{i}} x_{i j} \chi\left(E_{i j}\right)\right\}$. Then $f \in C$ and $\int_{X} d G \cdot f$ does not exist. This latter assertion can be verified as follows. Let $\left[K_{p} ; p \leqq m\right]$ be a partition of $X$ in $S$. There exists $p \leqq m$ and a subsequence $\left\{i_{k}\right\}$ such that $\left\|\sum_{j<n_{i_{k}}} G\left(E_{i_{k} j} \cap K_{p}\right)\left(x_{i_{k} j}\right)\right\|>i_{k}^{2} / m$. Let $y_{k j} \in E_{i_{k} j} \cap K_{p}$ if $E_{i_{k j}} \cap K_{p} \neq \varnothing$ and let $H_{k}=K_{p}-U_{j<n_{i_{k}}} E_{i_{k} j}$. Then (recall that $G\left(E_{i_{k} j} \cap K_{p}\right)=0$ if $\left.E_{i_{k} j} \cap K_{p}=\varnothing\right) \| G\left(K_{p}\right)\left(f\left(y_{q j}\right)\right)$ - $\left\{\sum_{j<n_{i k}} G\left(E_{i_{k} j} \cap K_{p}\right)\left(f\left(y_{k j}\right)\right)+G\left(H_{k}\right)\left(f\left(y_{q j}\right)\right)\right\} \|>\left(i_{k}^{2} / m\right)\left(1 / i_{k}\right)$ - $\left\|f\left(y_{q j}\right)\right\|\left(\left\|G\left(K_{p}\right)\right\|+\left\|G\left(H_{k}\right)\right\|\right)>i_{k} / m$ provided we choose $q$ to be sufficiently large.

A slight modification of this latter proof yields the following

THEOREM 3. If $g$ is a real valued finitely additive set function on $S$, then $g$ is bounded if, and only if, $\int_{X} f d g$ exists for each $f \in C$.

The dual of Theorem 3 is an immediate consequence of the proof given in [1] for the case where $B$ is the space of real numbers; how ${ }^{-}$ ever, the dual of Theorem 2 is not, in general, true-even, as the following example shows, when $\left(B, B_{1}\right)=B^{*}$. In view of examples (cf. $[5 ; 7 ; 2])$ of "wild" vector valued functions that are integrable, perhaps this latter phenomenon is not surprising.

Example 1. Suppose $X$ is the set of positive integers, $S$ is the algebra of finite subsets of $X$ and their complements, $B$ is the Banach space (sup norm) of bounded functions on $X,\left\{r_{i}\right\}$ is an enumeration of the rational numbers in $(0,1]$ and $f(i)=r_{i} \chi([i])$. Then $f$ is a $B$ valued function on $X$ that is discontinuous with respect to $S$. If $u \in B^{*}$ and $j$ is a positive integer, then we denote by $u_{j}$ the element of $B^{*}$ such that if $h \in B$, then $u_{j}(h)=h(j) u(\chi([j]))$; we also let $u_{0}=u$ $-\sum_{j \geq 1} u_{j}$. Then $\|u\|=\sum_{j \geq 0}\left\|u_{j}\right\|$. Let $G \in H\left(B^{*}\right)$ and let $t_{i}=G([i])$. Then $t_{0}=G([X])-\lim _{n \rightarrow \infty} \sum_{i \leq n} t_{i}$ exists and $\|G\|=\sum_{i \geq 0}\left\|t_{i}\right\|$. Let 
$\epsilon>0$. Then there exists a positive integer $n$ such that $\sum_{i>n}\left\|t_{i}\right\|$ $+\sum_{j>n}\left\|t_{0 j}\right\|<\epsilon / 2$. Let $\pi$ consist of the sets [1], $\cdots,[n],[k ; k>n]$.

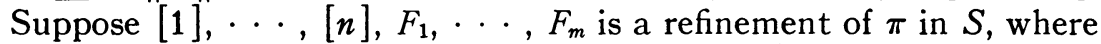
$F_{k}$ is finite if $k<m$ and $i_{k} \in F_{k}$. Then $\left|\sum_{k_{s} m} G\left(F_{k}\right)\left(f\left(i_{k}\right)\right)\right|$ $\leqq \sum_{k \leqq m}\left|G\left(F_{k}\right)\left(f\left(i_{k}\right)\right)\right|<\sum_{k<m}\left\|G\left(F_{k}\right)\right\|+\left|G\left(F_{m}\right)\left(f\left(i_{m}\right)\right)\right| \leqq \sum_{k>n}\left\|t_{k}\right\|$ $+r_{i_{m}}\left\|t_{0 i_{m}}\right\|<\epsilon / 2$. Hence $\int_{X} d G \cdot f$ exists.

In view of Example 1, a strong restriction on the range of $f$ is necessary in order to establish an analog of (a) in the case where the integrator is not real valued. To require that the range of $f$ be partitionable: for each $\epsilon>0$, there exists a partition $\pi$ of $X$ (not necessarily in $S$ ) such that $\sup _{E \in \pi} 0(f, E)<\epsilon$, provides such a restriction.

THEOREM 4. If $f$ is a partitionable B-valued function on $X$, then $f$ is continuous with respect to $S$ if, and only if, $\int_{X} d G \cdot f$ exists for each $G \in H\left(B^{*}\right)$.

Proof. Suppose $f$ is not continuous with respect to $S$. Then there exists $\epsilon>0$ such that if $\pi$ is a partition of $X$ in $S$, then $\sup _{E \in \pi} 0(f, E)$ $>\epsilon$. Let $\left[F_{i} ; i \leqq m\right]$ be a partition of $X$ such that $\sup _{i \leqq m} 0\left(f, F_{i}\right)$ $<\epsilon / 8$. There exist two integers $i$ and $j$ such that if $\pi$ is a partition of $X$ in $S$, then there exists $E \in \pi$ such that $E \cap F_{i} \neq \theta, E \cap F_{j} \neq \theta$ and $\inf _{x \in F_{i}, y \in F_{j}}\|f(x)-f(y)\|>3 \epsilon / 4$. Let $J=[F \in S$; there exists a partition $\pi$ of $F$ in $S$ such that if $K \in \pi$ then at least one of $K \cap F_{i}$ and $\left.K \cap F_{j}=\theta\right]$. Then $J$ is a proper ideal in $S$ and, hence, there exists a maximal proper ideal $M$ in $S$ that contains $J$. Let $x_{i} \in F_{i}$ and $x_{j} \in F_{j}$. There exists $L \in B^{*}$ such that $\|L\|=1$ and $L\left(f\left(x_{i}\right)-f\left(x_{j}\right)\right) \geqq 3 \epsilon / 4$. Let $G$ be the element of $H\left(B^{*}\right)$ such that if $E \in M$ then $G(E)=0$ and if $E \notin M$ then $G(E)=L$. Let $\pi=\left[E_{i} ; i \leqq n\right]$ be a partition of $X$ in $S$. Since $M$ is a maximal proper ideal in $S$ there exists uniquely an element $E_{k}$ of $\pi$ such that $E_{k} \notin M$ and, hence, if $u_{i} \in E_{i}$ for $i \leqq n$ then $\sum_{i \leqq n} G\left(E_{i}\right)\left(f\left(u_{i}\right)\right)=L\left(f\left(u_{k}\right)\right)$. However, since $E_{k} \notin M, E_{k} \cap F_{i} \neq \theta$ and $E_{k} \cap F_{j} \neq \theta$ and, hence, there exist $y_{i} \in E_{k} \cap F_{i}$ and $y_{j} \in E_{k} \cap F_{j}$. But,

$$
\begin{aligned}
& \left|L\left(f\left(y_{i}\right)-f\left(y_{i}\right)\right)\right| \\
& \quad \geqq\left|L\left(f\left(x_{i}\right)-f\left(x_{j}\right)\right)\right|-\left[\left|L\left(f\left(y_{i}\right)-f\left(x_{i}\right)\right)\right|+\left|L\left(f\left(x_{j}\right)-f\left(y_{j}\right)\right)\right|\right] \\
& \quad \geqq 3 \epsilon / 4-\|L\|\left[|| f\left(y_{i}\right)-f\left(x_{i}\right)\|+\| f\left(x_{j}\right)-f\left(y_{j}\right) \|\right]>\epsilon / 2
\end{aligned}
$$

and, hence, $\int_{x} d G \cdot f$ does not exist.

We wish to make one final remark concerning Example 1. Suppose $u \in B^{*}$. Then $u(f(j))=r_{j} u(\chi([j]))$. Since $\sum_{j}|u(\chi([j]))| \leqq\|u\|$, $\lim _{j} r_{j} u(\chi([j]))=0$ and, hence, $u f$ is a real valued function on $X$ that is continuous with respect to $S$. Hence $\int_{X}(u f) d g$ exists for each $g \in H(X, S)$. However, the range of $f$ is countable (hence separable) 
and there exist (many) elements $g$ of $H(X, S)$ such that $f$ is not measurable (cf. $[2 ; 3 ; 9]$ ) with respect to $g$. This phenomenon contrasts with the basic relation [9, Theorem 1.1] between weak measurability and measurability in the classical (sigma algebra, countably additive set function) case: in the classical case a necessary and sufficient condition that a function be measurable is that it be weakly measurable and separably valued.

\section{BIBLIOGRAPHY}

1. R. B. Darst, A note on abstract integration, Trans. Amer. Math. Soc. 99 (1961), 292-297.

2. - A continuity property for vector valued measurable functions, Pacific $\mathrm{J}$. Math. (to appear).

3. N. Dunford and J. T. Schwartz, Linear operators, Vol. 1, Interscience, New York, 1958.

4. M. Frechet, L'intégrale abstract d'une fonction abstraite d'une variable abstraite et son application d la moyenne d'un élément aléatoire de nature quelconque, Revue Sci. 82 (1944), 483-512.

5. L. M. Graves, Riemann integration and Taylor's theorem in general analysis, Trans. Amer. Math. Soc. 29 (1927), 163-177.

6. T. H. Hildebrandt, On bounded linear functional operations, Trans. Amer. Math. Soc. 36 (1934), 868-875.

7. - Integration in abstract spaces, Bull. Amer. Math. Soc. 59 (1953), 111-139.

8. S. Leader, On universally integrable functions, Proc. Amer. Math. Soc. 6 (1955), $232-234$.

9. B. J. Pettis, On integration in vector spaces, Trans. Amer. Math. Soc. 44 (1938), 277-304.

Massachusetts Institute of Technology 